\title{
Inhibition of autophagy promotes cell apoptosis induced by the proteasome inhibitor MG-132 in human esophageal squamous cell carcinoma EC9706 cells
}

\author{
DONGLEI LIU $^{1 *}$, MIN GAO $^{2 *}$, YANG YANG $^{1}, \mathrm{YU}^{1}{ }^{1}, \mathrm{KAI} \mathrm{WU}^{1}$ and SONG ZHAO ${ }^{1}$ \\ ${ }^{1}$ Department of Thoracic Surgery; ${ }^{2}$ Intensive Care Unit, The First Affiliated Hospital of Zhengzhou University, \\ Zhengzhou, Henan 450052, P.R. China
}

Received June 20, 2014; Accepted February 27, 2015

DOI: $10.3892 / \mathrm{ol} .2015 .3047$

\begin{abstract}
Lysosome-dependent macroautophagy, also termed autophagy, and the ubiquitin-proteasome system and are the primary intracellular pathways involved in protein degradation. Previous studies have demonstrated that proteasome inhibitors are able to inhibit tumor growth and activate autophagy. The present study investigated the effect of the proteasome inhibitor MG-132 on cellular proliferation using a cell counting kit 8 assay, and the effect of the agent on apoptosis and autophagy was assessed using flow cytometry and monodansylcadaverine, respectively. Western blot analysis was used to investigate protein changes during the course of treatment. It was revealed that MG-132 inhibited cell proliferation, activated autophagy and induced cell death in EC9706 cells. Autophagy was activated through the class III PI3K pathway, and the expression of the Beclin-1 protein was determined to be significantly upregulated. However, the autophagy inhibitor 3-methyladenine (3-MA) inhibited the expression of the autophagy-associated protein Beclin-1 and reduced the accumulation of autophagic vacuoles induced by MG-132. MG-132-induced apoptosis was enhanced by the autophagy inhibitor 3-MA, which may be a result of caspase-3 activation in the EC9706 cells. These findings suggest that inhibition of the proteasome can induce autophagy in human ESCC cells, and also increase cell death. This indicates that proteasome inhibitors may be potential novel anti-cancer agents for the adjuvant treatment of esophageal squamous cell carcinoma.
\end{abstract}

Correspondence to: Dr Song Zhao, Department of Thoracic Surgery, The First Affiliated Hospital of Zhengzhou University, 1 Jiansge East Road, Zhengzhou, Henan 450052, P.R. China

E-mail: zhaosong@zzu.edu.cn

*Contributed equally

Key words: proteasome inhibitor, autophagy, esophageal squamous carcinoma cells

\section{Introduction}

The ubiquitin-proteasome system (UPS) and lysosome-dependent macroautophagy, also termed autophagy, are the primary conserved intracellular pathways involved in protein degradation. These pathways work together in order to maintain homeostasis in eukaryotic cells (1). UPS-mediated proteolysis affects several different proteins through proteasome-mediated degradation, which is involved in the regulation of the cell cycle, apoptosis and cellular differentiation (2). Therefore, targeting this pathway using proteasome inhibitors may represent a novel approach for the treatment of cancer (3). A number of previous studies have demonstrated that proteasome inhibitors can induce tumor cell death via inhibiting proteasome activity $(4,5)$.

Autophagy is an evolutionarily conserved intracellular mechanism that degrades long-lived, misfolded proteins and damaged organelles in order to maintain cellular homeostasis by providing substrates and recycling amino acids and nucleotides (6). Although autophagy can be activated in a number of various cancer cells, including esophageal cancer, by different approaches, such as chemoradiotherapy (7), the exact role of autophagy in cancer cells is complex. In certain circumstances, autophagy demonstrates a protective role in cancer cells, whereas in others, it is involved in type II programmed cell death, termed autophagic cell death (8). One previous study reported that when UPS is inhibited, autophagy is upregulated (9). This suggests that the UPS and autophagy may act as two compensatory mechanisms that modulate protein degradation.

Esophageal carcinoma is the eighth most common cause of cancer-associated mortality worldwide (10). Esophageal squamous cell carcinoma (ESCC) is the main subtype in developing countries, particularly in China (11). At the time of diagnosis, a large proportion of patients with ESCC have lost the optimum opportunity for surgery. The five-year survival rate for ESCC remains extremely low as a result of resistance to anti-cancer therapies, including chemotherapy and radiotherapy (12). Previous studies have indicated that the inhibition of autophagy can potentiate chemo-radiotherapy-induced apoptosis in ESCC cells $(13,14)$. Due to the fact that the proteasome inhibitor has become a novel target for cancer 
therapy, the present study investigated whether autophagy could be activated by the inhibition of the proteasome in the ESCC cells. Furthermore, the ability of the inhibition of autophagy to enhance proteasome inhibitor-induced ESCC cell death was also investigated.

\section{Materials and methods}

Cell lines and culture. The poorly-differentiated ESCC EC9706 cell line was purchased from the State Key Laboratory of Molecular Oncology, Chinese Academy of Medical Sciences (Beijing, China). First, the EC9706 cells were cultured in RPMI-1640 medium (Gibco Life Technologies, Carlsbad, CA, USA) containing 10\% fetal serum (FBS) (GE Healthcare Life Sciences, Logan, UT, USA), $100 \mu \mathrm{g} / \mathrm{ml}$ streptomycin and 100 units $/ \mathrm{ml}$ penicillin in a humidified $5 \% \mathrm{CO}_{2}$ atmosphere at $37^{\circ} \mathrm{C}$. The medium was changed every two days. The cells were assigned to the control, MG-132 and MG-132 plus 3-methyladenine (3-MA) groups. Subsequent to a 24-h incubation, the culture medium was replaced with fresh medium containing no additional reagents for the control group, MG-132 for the MG-132 group, or a combination of MG-132 and 3-MA for the MG-132 plus 3-MA group. The EC9706 cells were then treated, depending on the group, with $20 \mu \mathrm{M}$ MG-132, $5 \mathrm{mM}$ 3 -MA or the two combined for $24 \mathrm{~h}$.

Chemical reagents. The MG-132 was purchased from EMD Millipore(Billerica,MA,USA).First, the MG-132 was dissolved in phosphate-buffered saline (PBS) to a storage concentration of $50 \mathrm{mM}$. Next, 3-MA (Sigma-Aldrich, St. Louis, MA, USA) was dissolved in PBS to generate a $100 \mathrm{mM}$ stock solution and was maintained at room temperature until use. The subsequent dilution was made using RPMI-1640 medium in order to create the desired concentration. Monodansylcadaverine (MDC; Sigma-Aldrich) was used to assess autophagy. The antibodies against Beclin-1 and caspase- 9 were purchased from Santa Cruz Biotechnology, Inc. (Dallas, TX, USA).

Measurement of cell viability and apoptosis. Cell viability was detected using the cell counting kit 8 (CCK-8) assay. First, the cells were seeded into a 96-well flat bottom microplate at a density of $1 \times 10^{5}$ cells in $100 \mu 1$ per well. Subsequent to treatment with MG-132, 3-MA or the two combined, $100 \mu 1$ medium was replaced with an equal volume of fresh medium containing 10\% CCK-8 (WST-8; Dojindo Laboratories, Tokyo, Japan). Next, the cells were incubated for $3 \mathrm{~h}$ at $37^{\circ} \mathrm{C}$, and the absorbance of the solution was analyzed at $450 \mathrm{~nm}$ using a microplate spectrophotometer (BioTek EL 340; BioTek Instruments, Inc., Winooski, VT, USA). The cell viabilities were then calculated using the following equation:

$$
\text { Cell viability }(\%)=\left(1-\mathrm{A}_{450 \text { sample }} / \mathrm{A}_{450 \text { control }}\right) \times 100
$$

Each experiment was performed in triplicate.

Cell apoptosis was detected by flow cytometry. Subsequent to treatment, the attached and floating cells were harvested and washed twice with PBS. Next, $5 \mu \mathrm{l}$ Annexin V-fluorescein isothiocyanate (FITC) and $5 \mu \mathrm{l}$ propidium iodide (PI) were added according to the instructions of the Annexin V-FITC Apoptosis Detection kit (Nanjing KeyGen Biotech Co., Ltd.,
Nanjing, China). The cells were then incubated in the dark at room temperature for $15 \mathrm{~min}$. Finally, the cells were analyzed using a flow cytometry system (FACScan; BD Biosciences, San Jose, CA, USA) and the data were analyzed using Cell Quest software (BD Biosciences).

Analysis of autophagy using MDC. In order to measure the autophagic ratio, the EC9706 cells were plated into 24-cell plates at a density of $1 \times 10^{5}$ cells. Subsequent to a 24 -h incubation with the different drugs, the cell pellets were suspended with $0.05 \mathrm{mM} \mathrm{MDC}$ for $60 \mathrm{~min}$ at $37^{\circ} \mathrm{C}$, washed with PBS three times, fixed with $4 \%$ paraformaldehyde for $15 \mathrm{~min}$ at $4^{\circ} \mathrm{C}$ and then collected in $10 \mathrm{mM}$ Tris- $\mathrm{HCl}(\mathrm{pH} 8.0)$ containing $0.1 \%$ Triton X-100. An inverted fluorescence microscope (Olympus IX70; Olympus, Tokyo, Japan) was then used to identify changes in the appearance of the autophagic vacuoles and subsequently capture images. The fluorescence intensity of the cells in the different groups was measured by flow cytometry.

Western blotting. Subsequent to treatment with MG-132 alone or in combination with the autophagic inhibitor 3-MA, the EC9706 cells were harvested from cultured dishes and lysed in cold lysis buffer for $20 \mathrm{~min}$. The cell extracts were then collected and centrifuged for $5 \mathrm{~min}$ at 9,180 x g. Overall, $20 \mu \mathrm{g}$ total protein obtained from the whole cell lysates were boiled in $1 \mathrm{X}$ SDS buffer for $5 \mathrm{~min}$, separated by $12 \%$ SDS-PAGE, and then electrotransferred using a semi-dry transfer method to nitrocellulose membranes (GE Healthcare Life Sciences, Uppsala, Sweden). Following electrophoretic transfer, the membrane was blocked at $4^{\circ} \mathrm{C}$ overnight. Subsequent to blocking, the membranes were incubated with the primary antibodies at the recommended concentrations for $1 \mathrm{~h}$. The The rabbit polyclonal anti-human antibodies against caspase-3, Beclin-1, and $\beta$-actin were obtained from Santa Cruz Biotechnology, Inc. Next, the membranes were incubated with the anti-mouse immunoglobulin $\mathrm{G}$ horseradish peroxidase-conjugated secondary antibodies (Santa Cruz Biotechnology, Inc.). Finally, the blots were developed using enhanced chemiluminescence (GE Healthcare Life Sciences, Piscataway NJ, USA) on Kodak X-omat LS film (Kodak, Rochester, NY, USA) and the densitometry was performed using Kodak 1D Image Analyses software, version 3.5 (Kodak).

Statistical analysis. All data represent at least three independent experiments and are expressed as the mean \pm standard deviation. Student's t-test was used for the statistical analyses. $\mathrm{P}<0.05$ was considered to indicate a statistically significant difference.

\section{Results}

Proteasome inhibitor MG-132 inhibits cell proliferation and induces cell death in ESCC cells. After the EC9706 cells were treated with various concentrations of MG-132 or 3-MA for $48 \mathrm{~h}$, the CCK-8 was used to assess cell viability. As shown in Fig. 1, EC9706 cell growth was effectively inhibited by MG-132 or 3-MA in a dose- and time-dependent manner. The half maximal inhibitor concentration of MG-132 at $48 \mathrm{~h}$ was $20 \pm 2.1 \mu \mathrm{mol} / 1$. Therefore, $20 \mu \mathrm{mol} / 1 \mathrm{MG}-132$ was 
selected for further experiments. In addition, it was revealed that MG-132 significantly inhibited the proliferation of EC9706 cells. By contrast, the rates of cell proliferation were significantly reduced in the MG-132 and 3-MA combined treatment group.

3-MA reverses $M G$-132-induced autophagy in the ESCC cell line. In order to determine the effect of proteasome inhibition on autophagy, the fluorescence of MDC was used to observe changes in the appearance of the autophagic vacuoles (15). As shown in Fig. 2, there was no significant difference in the autophagy vacuoles in the 3-MA-treated group compared with the control group. However, the number of autophagic vacuoles stained by MDC in the MG-132 group was markedly higher compared with the control group. Following the addition of 3-MA, the number of vacuoles was significantly decreased compared with the cells treated with MG-132 alone. Furthermore, compared with the MG-132 group, the fluorescence intensity was decreased and the vacuoles were reduced in the 3-MA and MG-132 combined group.

In order to investigate the underlying mechanism by which the proteasome inhibitor MG-132 induces autophagy, western blot analysis was performed. Beclin-1 is an important regulator that promotes autophagy, and is also associated with a number of biological processes, including development, immunity, adaptation to stress, tumorigenesis, endocytosis, cytokinesis, aging and cell death (16). As shown in Fig. 3, the expression of Beclin-1 was significantly upregulated in the MG-132 group. However, following the addition of 3-MA, the expression of Beclin-1 was markedly inhibited. This indicates that autophagy induced by MG-132 can be reversed by co-treatment with 3-MA in EC9706 cells.

MG-132-induced cell death increased by 3-MA in the ESCC cell line. CCK-8 assays were performed in order to investigate the effect of autophagy inhibition on cell viability. As shown in Fig. 4, 5 mmol/l 3-MA significantly enhanced the inhibition of cell viability induced at $24 \mathrm{~h}$ by MG-132. This suggests that autophagy inhibition can effectively inhibit cell viability. In addition, the Annexin V-FITC and PI staining assay was used to observe the apoptosis of ESCC cells treated with MG-132. As shown in Fig. 4, the apoptosis rate of the EC9706 cells treated with MG-132 in combination with 3-MA significantly increased between 57.47 and $79.40 \%$ compared with the group treated with MG-132 alone. Next, expression of the apoptosis-associated protein caspase-3 was examined. Fig 5 shows that 3-MA increased the levels of caspase-3 induced by MG-132. Together, these results indicate that the inhibition of autophagy is able to increase MG-132-induced apoptosis in ESCC cells.

\section{Discussion}

Chemotherapy is widely used for the treatment of patients with metastatic or unresectable ESCC (17). However, ESCC cells have developed resistance to chemotherapeutic drugs, which has resulted in a reduction in the five-year survival rate. Therefore, a requirement exists to identify novel therapeutic strategies or adjuvant drugs for patients with ESCC. The present study revealed that inhibition of the proteasome by

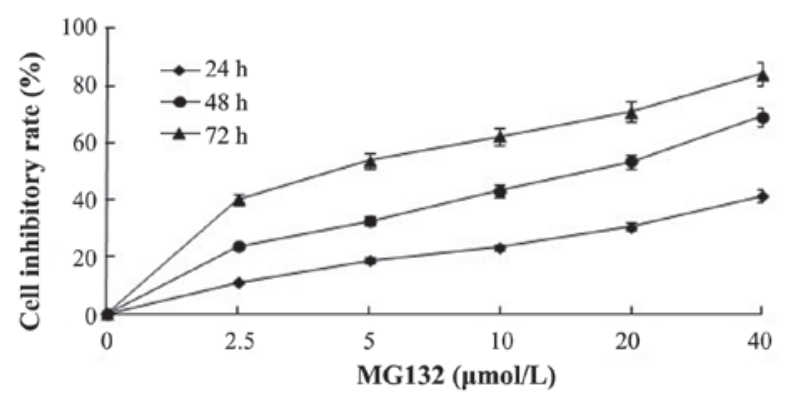

Figure 1. The proteasome inhibitor MG-132 significantly decreased the cell viability in EC9706 cells in a dose- and time-dependent manner.

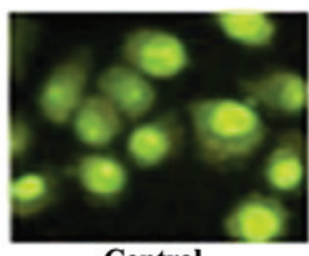

Control

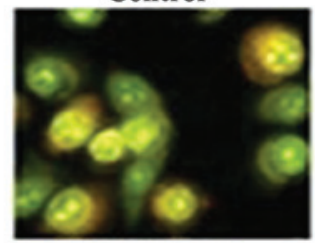

MG-132

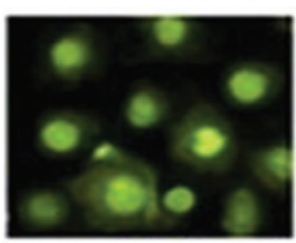

3-MA

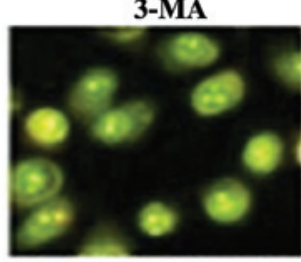

MG-132+3-MA

\section{Incorporation} (absorptance / cell number)

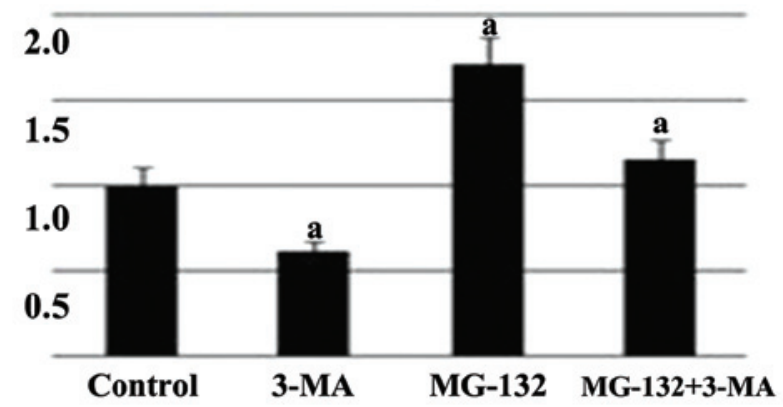

Figure 2. Increased autophagy induced by MG-132 is inhibited by 3-MA. The autophagic vacuoles were observed with MDC. In the MG-132 group, the autophagic vacuoles in the EC9706 cells were increased compared with those in the control group. However, this increase was inhibited by the addition of 3-MA. ${ }^{\text {aP }}<0.05$ vs. the control group. 3-MA, 3-methyladenine; MDC, monodansylcadaverine.

MG-132 decreased cell proliferation, induced cell death and activated autophagy in EC9706 cells. Furthermore, the results demonstrated that MG-132 inhibited the proliferation in EC9706 cells in dose- and time-dependent manner.

The proteasome system and autophagy machinery are regarded as the two major cellular protein degradation systems (3). It has been identified that proteasome inhibitor-induced autophagy is able to control endoplasmic reticulum stress and reduce cell death in cancer cells by activating the downstream inositol-requiring enzyme-1/c-Jun NH2-terminal 

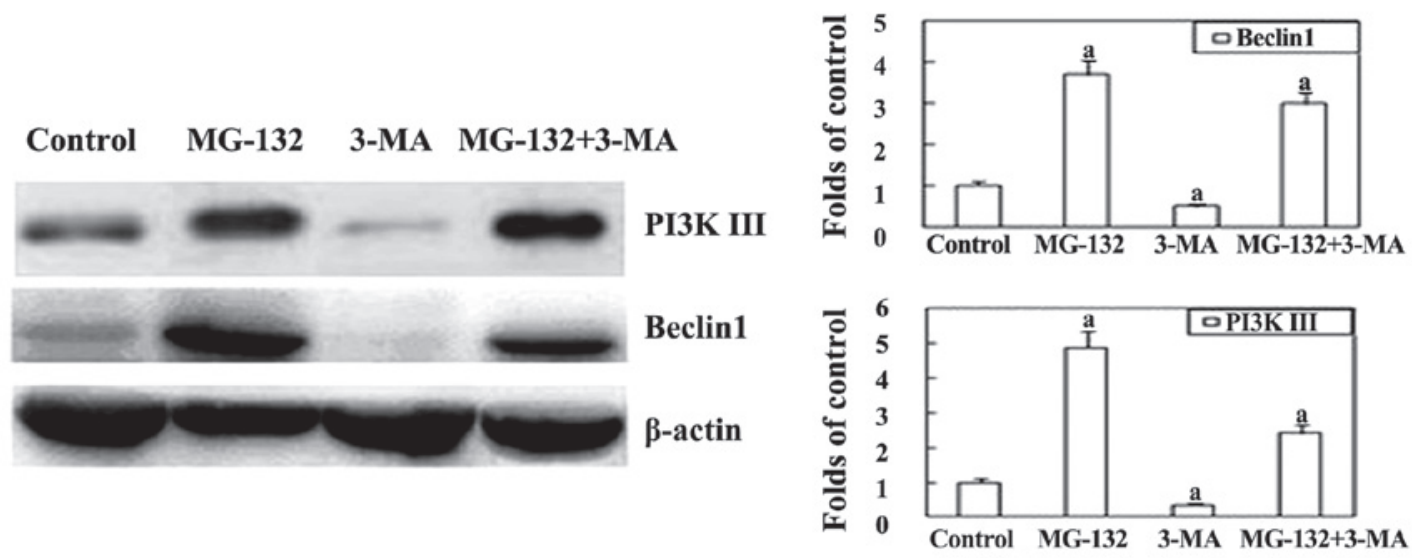

Figure 3. Relative expression of autophagy-associated proteins in the EC9607 cells. Beclin-1 and PI3K III were upregulated in the MG-132 group. The addition of 3-MA counteracted the upregulation of Beclin-1 and PI3K III. ${ }^{a} \mathrm{P}<0.05$ vs. the control group. 3-MA, 3-methyladenine.
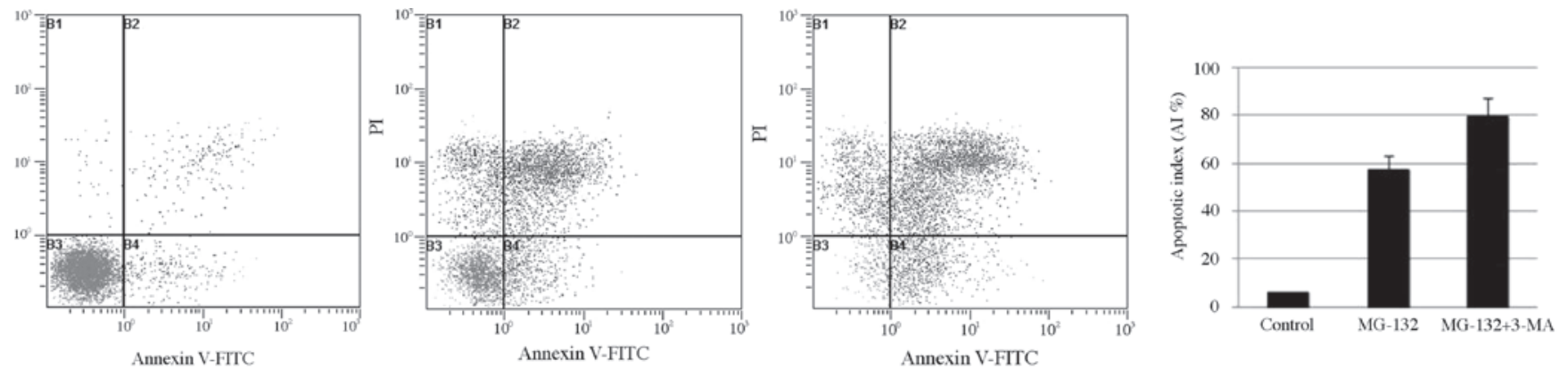

Figure 4. Flow cytometry results revealing that 3-MA increased the MG-132-induced apoptosis of EC9706 cells. Compared with the MG-132 group, the apoptosis rate of the EC9706 cells that were treated with MG-132 in combination with 3-MA was significantly higher $(\mathrm{P}<0.05)$. 3-MA, 3-methyladenine.
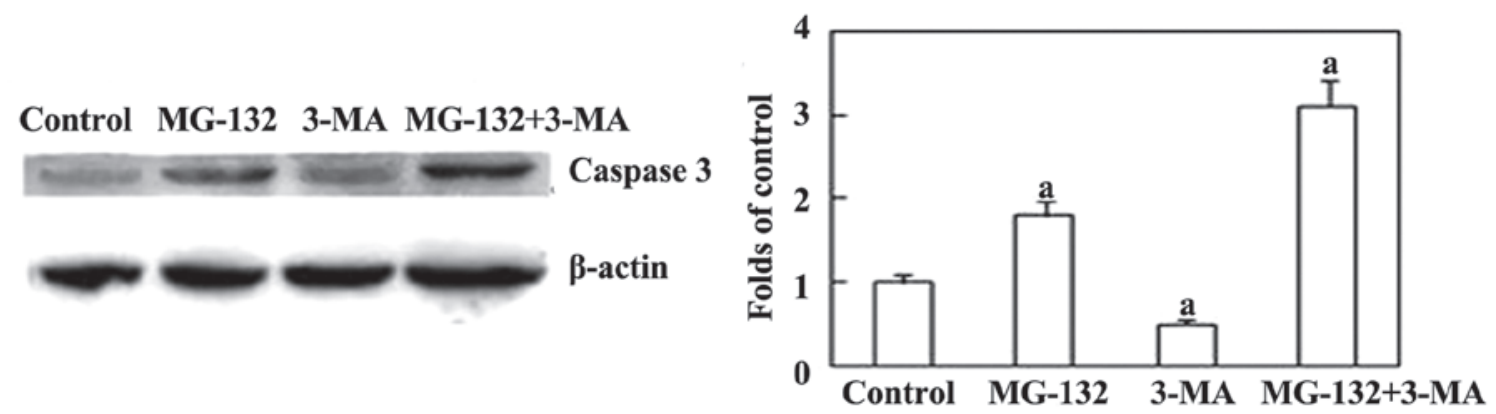

Figure 5. Western blotting results revealing the effect of 3-MA on protein expression in the MG-132-treated EC9607 cells. For the graphs, the results were calculated and then presented as the mean \pm standard error of the mean from the three independent experiments. ${ }^{\text {a }}<0.05$ vs. the control group. 3 -MA, 3-methyladenine.

kinase pathway (18). In the present study, autophagy was assessed using biochemical methods, including MDC and western blotting. As the results demonstrate, the expression of the autophagy-associated protein, Beclin-1, was significantly upregulated in EC9706 cells following a 48-h incubation with MG-132.

A number of studies have established that autophagy can be activated in a variety of cancer cells under different circumstances, including chemo-radiotherapy (9). However, the precise role of autophagy in tumor cell death and survival remains unclear. In order to investigate the role of autophagy in MG-132-induced EC9706 cell apoptosis, the present study used the autophagy inhibitor, 3-MA, a class III phosphatidylinositol 3-kinase inhibitor and a specific inhibitor of autophagy. MG-132-induced autophagy in EC9706 cells was significantly inhibited by the addition of 3-MA. Furthermore, the inhibition of autophagy enhanced MG-132-induced apoptosis in EC9706 cells through caspase-3 activation. This suggests that caspase- 3 may be the primary protease involved in the apoptosis pathways (19). These findings indicate that autophagy may be utilized as a protective mechanism against cell death in MG-132-induced EC9706 cells, and that its inhibition may enhance the EC9706 cell death induced by MG-132. 
There are two important signaling pathways involved in the process of autophagy, namely the PI3K/AKT/mTOR and class III PI3K pathways. The specific inhibitor of autophagy, 3-MA, is known to work by inhibiting the Beclin-1-PI3K III complex, which is a component important for the formation of autophagosomes (20). The present study revealed that following the addition of 3-MA, the expression of the PI3K III protein was lower compared with that of the MG-132 group. These results indicate that MG-132-induced autophagy in EC9706 cells may be activated through the class III PI3K pathway.

In conclusion, the results of the present study suggest that the proteasome inhibitor, MG-132, induces cell growth inhibition and cell death in EC9706 cells. In addition, they demonstrate that inhibition of the proteasome activates the process of autophagy, and that MG-132-induced apoptosis is enhanced by autophagy inhibition through the activation of the class III PI3K pathway and the release of caspase-3. These findings suggest that proteasome inhibitors may be potential novel anti-cancer agents for the adjuvant treatment of ESCC.

\section{Acknowledgements}

This study was supported by the Youth Innovation Fund Project of the First Affiliated Hospital of Zhengzhou University and the Key Project of Science and Technology of the Education Department of Henan province (grant no. 14A320076).

\section{References}

1. Wu WK, Cho CH, Lee CW, Wu K, Fan D, Yu J and Sung JJ: Proteasome inhibition: a new therapeutic strategy to cancer treatment. Cancer Lett 293: 15-22, 2010.

2. Sorokin AV, Kim ER and Ovchinnikov LP: Proteasome system of protein degradation and processing. Biochemistry (Mosc) 74: 1411-1142, 2009.

3. Wojcik S: Crosstalk between autophagy and proteasome protein degradation systems: possible implications for cancer therapy. Folia Histochem Cytobiol 51: 249-264, 2013.

4. Orlowski RZ and Kuhn DJ: Proteasome inhibitors in cancer therapy: lessons from the first decade. Clin Cancer Res 14: 1649-1657, 2008.
5. Guo N and Peng Z: MG132, a proteasome inhibitor, induces apoptosis in tumor cells. Asia Pac J Clin Oncol 9: 6-11, 2013.

6. Mathew R, Karantza-Wadsworth V and White E: Role of autophagy in cancer. Nat Rev Cancer 7: 961-967, 2007.

7. Janku F, McConkey DJ, Hong DS and Kurzrock R: Autophagy as a target for anticancer therapy. Nat Rev Clin Oncol 8: 528-539, 2011.

8. Levine B and Kroemer G: Autophagy in the pathogenesis of disease. Cell 132: 27-42, 2008.

9. Wu WK, Sakamoto KM, Milani M, et al: Macroautophagy modulates cellular response to proteasome inhibitors in cancer therapy. Drug Resist Updat 13: 87-92, 2010.

10. Kamangar F, Dores GM and Anderson WF. Patterns of cancer incidence, mortality and prevalence across five continents: defining priorities to reduce cancer disparities in different geographic regions of the world. JClin Oncol 24: 2137-2150, 2006.

11. Pisani P, Parkin DM, Bray F and Ferlay J: Estimates of the worldwide mortality from 25 cancers in 1990. Int J Cancer 83: 18-29, 1999.

12. Hanahan D and Weinberg RA: Hallmarks of cancer: the next generation. Cell 144: 646-674, 2011.

13. Liu D, Yang Y, Liu Q and Wang J: Inhibition of autophagy by 3-MA potentiates cisplatin-induced apoptosis in esophageal squamous cell carcinoma cells. Med Oncol 28: 105-111,2011.

14. Chen YS, Song HX, Lu Y, et al: Autophagy inhibition contributes to radiation sensitization of esophageal squamous carcinoma cells. Dis Esophagus 24: 437-443, 2011.

15. Biderbik A, Kern HF and Elsässer HP. Monodansylcadaverine (MDC) is a specific in vivo marker for autophagic vacuoles. Eur J Cell Biol 66: 3-14, 1995.

16. Wirawan E, Lippens S, Vanden Berghe T, Romagnoli A, Fimia GM, Piacentini M, Vandenabeele P: Beclin1: a role in membrane dynamics and beyond. Autophagy 8: 6-17, 2012.

17. van Hagen P, Hulshof MC, van Lanschot JJ, et al; CROSS Group: Preoperative chemoradiotherapy for esophageal or junctional cancer. N Engl J Med 366: 2074-2084, 2012.

18. Ding WX, Ni HM, Gao W, Yoshimori T, Stolz DB, Ron D and Yin XM: Linking of autophagy to ubiquitin-proteasome system is important for the regulation of endoplasmic reticulum stress and cell viability. Am J Pathol 171: 513-524, 2007.

19. Harrington HA, Ho KL, Ghosh S and Tung KC: Construction and analysis of a modular model of caspase activation in apoptosis. Theor Biol Med Model 5: 26, 2008.

20. Wu YT, Tan HL, Shui G, et al: Dual role of 3-methyladenine in modulation of autophagy via different temporal patterns of inhibition on class I and III phosphoinositide 3-kinase. J Biol Chem 285: 10850-10861, 2010. 